\title{
Operator approach to complexity: Excited states
}

\author{
Wung-Hong Huang $\oplus^{*}$ \\ Department of Physics, National Cheng Kung University, No. 1, University Road, Tainan City 701, Taiwan
}

(Received 13 May 2019; published 17 September 2019)

\begin{abstract}
We evaluate the complexity of the free scalar field by the operator approach in which the transformation matrix between the second quantization operators of the reference state and target state is regarded as the quantum gate. We first examine the system in which the reference state is two noninteracting oscillators with the same frequency $\omega_{0}$ while the target state is two interacting oscillators with frequency $\tilde{\omega}_{1}$ and $\tilde{\omega}_{2}$. We calculate the geodesic length on the associated group manifold of gate matrix and reproduce the known value of ground-state complexity. Next, we study the complexity in the excited states. Although the gate matrix is very large we can transform it to a diagonal matrix and obtain the associated complexity. We explicitly calculate the complexity in several excited states and prove that the square of the geodesic length in the general state $|\mathrm{n}, \mathrm{m}\rangle$ is $D_{(\mathrm{n}, \mathrm{m})}^{2}=(n+1)\left(\ln \sqrt{\frac{\tilde{\omega}_{1}}{\omega_{0}}}\right)^{2}+(m+1)\left(\ln \sqrt{\frac{\tilde{\omega}_{2}}{\omega_{0}}}\right)^{2}$. The results are extended to the $N$ couple harmonic oscillators which correspond to the lattice version of the free scalar field.
\end{abstract}

DOI: $10.1103 /$ PhysRevD.100.066013

\section{INTRODUCTION}

Complexity plays an important role in understanding how spacetime emerges from field theory degrees of freedom within the AdS/CFT correspondence [1-4], besides the entanglement entropy [5]. It relates to the tensor network models and involves the dynamics of black hole interiors [6]. In the context of the eternal AdSSchwarzchild black hole the wormhole which connects the two sides grows linearly with time [7], which is conjectured to dual to the growth of complexity of the dual CFT state [8-10].

In the context of AdS/CFT two interesting proposals are used to evaluate the holographic complexity. The first is complexity $=$ volume $(\mathrm{CV})$ conjecture [11], which suggests that complexity is dual to the volume of an extremal (codimension one) bulk surface anchored to a certain time slice in the boundary. The second is complexity $=$ action (CA) conjecture [12-14] which identifies the complexity with the gravitational action evaluated on a particular bulk region, known as the Wheeler-DeWitt (WDW) patch. Complexity is the number of operations $\left\{\mathcal{O}^{I}\right\}$ needed to transform a reference state $\left|\psi_{R}\right\rangle$ to a target state $\left|\psi_{T}\right\rangle$. The operators are called quantum gates and the more gates we need the more complex the target state. We can define the

\footnotetext{
*whhwung@mail.ncku.edu.tw
}

Published by the American Physical Society under the terms of the Creative Commons Attribution 4.0 International license. Further distribution of this work must maintain attribution to the author(s) and the published article's title, journal citation, and DOI. Funded by SCOAP. affine parameter " $s$ " associated to an unitary operator $U(s)$ and use a set of function $Y^{I}(s)$ to characterize the quantum circuit. The unitary operation that connects the reference state and target state is

$$
\begin{gathered}
U(s)=\overrightarrow{\mathcal{P}} e^{-\int_{0}^{s} Y^{I}(s) \mathcal{O}^{I}}, \quad\left|\psi_{R}\right\rangle=U(0)\left|\psi_{R}\right\rangle, \\
\left|\psi_{T}\right\rangle=U(1)\left|\psi_{R}\right\rangle,
\end{gathered}
$$

where $\overrightarrow{\mathcal{P}}$ indicates a time ordering along $s$. The circuit depth $D[U]$, which is also called the cost function, is defined by

$$
D[U]=\int_{0}^{1} d s \sqrt{\sum_{I}\left|Y^{I}(s)\right|^{2}} .
$$

Using it, the complexity $\mathcal{C}$ is defined by

$$
\mathcal{C}=\operatorname{Min}_{\left\{\mathrm{Y}^{\mathrm{I}}\right\}} D[U] .
$$

For the models that have been studied the quantum gates are the group generators. Thus the minimum in $\mathcal{C}$ means that we are to calculate the geodesic in the Riemann space of the group manifold.

The calculation along this line was initial in Refs. [15,16], which considered the free scalar field. Later, it was extended to study the free fermions [17-19], quenched system [20-22], coherence state [23], and interacting model [24]. The investigation in Ref. [15] showed that once the cost function is chosen to be

$$
D[U]=\int_{0}^{1} d s \sum_{I}\left|Y^{I}(s)\right|^{2}
$$


the field theory calculation could match to the gravity method.

The most studies in field theory are considering the Gaussian ground state $[15,17,18]$ in the free field or exponential type wave function in the interacting model [24], which are reviewed in the next section.

In this paper we will present the operator approach to evaluate the complexity in free scalar field theory. Note that the operator approach has been used in Refs. [16,18,20,21], which study the fermion theory and quench mode. In these papers the reference operator and target operator are the creation operators with different mass or frequency. The Bogoliubov transformations between the operators relate to the quantum gate of the theory. In our study the reference operator is the creation operator of the noninteracting harmonic oscillator while the target operator is that in the interacting harmonic oscillator. We regard the transformation between the create operators of the reference state and the target state as the quantum gate and calculate the geodesic length on the associated group manifold. Since in the operator approach we need not to use the explicit form of the wave function, we can study the complexity in the excited states. Note that the excited-state wave function of harmonic oscillation is not the pure exponential form and the wave function approach is hard to work, as commented on in Sec. II.

The paper is organized as follows. In Sec. II, we quickly review the method in Ref. [15]. After describing the lattice free scalar field as coupled harmonic oscillators we consider first the 2 coupled oscillator system. We review how the transformation between the ground-state wave function of the reference state (i.e., two noninteracting oscillators) and the target state (i.e., two interacting oscillators) can be used to calculate the complexity of the ground state. In Sec. III, we setup the notation and describe the transformation between the second quantization operators in the reference state and target state. We find that the gate matrix can be reduced to diagonal form after orthogonal transformation. Then, we calculate circuit depth $D[U]$ and the complexity. The result reproduces the known value of ground-state complexity in Ref. [15].

In Sec. IV we carefully calculate the complexity in several excited states and prove that the square of geodesic length in the general state $|\mathrm{n}, \mathrm{m}\rangle$ is $D_{(\mathrm{n}, \mathrm{m})}^{2}=(n+1)\left(\ln \sqrt{\frac{\tilde{\omega}_{1}}{\omega_{0}}}\right)^{2}+$ $(m+1)\left(\ln \sqrt{\frac{\tilde{\omega}_{2}}{\omega_{0}}}\right)^{2}$. In Sec. V we describe how the calculations in previous sections, which are focused on two coupled harmonic oscillators, can be extended to $N$ coupled harmonic oscillators, which describes lattice free scalar field theory. A final summary appears in the last section. In Appendix A we discuss the relation of geodesic length between a gate matrix before and after being transformed to a diagonal form. In Appendix B we derive the summation formulas that are used to derive the complexity formula in the excited states.

\section{FREE SCALAR FIELD AND HARMONIC OSCILLATOR: WAVE FUNCTION APPROACH}

We consider the Hamiltonian of a free scalar field in $d$ spacetime dimensions

$$
H=\frac{1}{2} \int d^{d-1} x\left[\pi(x)^{2}+\vec{\nabla} \phi(x)^{2}+m^{2} \phi(x)^{2}\right] .
$$

Placing the theory on a square lattice with lattice spacing $\delta$ the Hamiltonian is described by a lattice version [15]

$$
\begin{aligned}
H= & \frac{1}{2} \sum_{\vec{n}}\left\{\frac{p(\vec{n})^{2}}{\delta^{d-1}}+\delta^{d-1}\left[\frac { 1 } { \delta ^ { 2 } } \sum _ { i } \left(\left(\phi(\vec{n})-\phi\left(\vec{n}-\hat{x}_{i}\right)\right)^{2}\right.\right.\right. \\
& \left.\left.+m^{2} \phi(\vec{n})^{2}\right]\right\},
\end{aligned}
$$

where $\hat{x}_{i}$ are unit vectors pointing along the spatial directions of the lattice. By redefining $X(\vec{n})=\delta^{d / 2} \phi(\vec{n})$, $P(\vec{n})=p(\vec{n}) / \delta^{d / 2}, M=1 / \delta, \omega=m$, and $\Omega=1 / \delta$ the lattice Hamiltonian becomes

$$
\begin{aligned}
H= & \sum_{\vec{n}}\left\{\frac{P(\vec{n})^{2}}{2 M}+\frac{1}{2} M\left[\omega^{2} X(\vec{n})^{2}+\Omega^{2} \sum_{i}(X(\vec{n})\right.\right. \\
& \left.\left.\left.-X\left(\vec{n}-\hat{x}_{i}\right)\right)^{2}\right]\right\},
\end{aligned}
$$

where $\omega=m$ and $\Omega=1 / \delta$. The resulting theory of free scalar field is essentially a quantum mechanical problem with an infinite family of coupled harmonic oscillators.

Consider first a simple case of two coupled harmonic oscillators:

$H=\frac{1}{2}\left[p_{1}^{2}+p_{2}^{2}+\omega^{2}\left(x_{1}^{2}+x_{2}^{2}\right)+\Omega^{2}\left(x_{1}-x_{2}\right)^{2}\right]$,

where $x_{1}, x_{2}$ label their spatial positions after setting $M_{1}=M_{2}=1$ for simplicity. The Hamiltonian expressed in terms of the normal modes is

$$
H=\frac{1}{2}\left(\tilde{p}_{1}^{2}+\tilde{\omega}_{1}^{2} \tilde{x}_{1}^{2}+\tilde{p}_{2}^{2}+\tilde{\omega}_{2}^{2} \tilde{x}_{2}^{2}\right)
$$

where

$$
\begin{gathered}
\tilde{x}_{\{1,2\}} \equiv \frac{1}{\sqrt{2}}\left(x_{1} \pm x_{2}\right), \quad \tilde{p}_{\{1,2\}} \equiv \frac{1}{\sqrt{2}}\left(p_{1} \pm p_{2}\right), \\
\left\{\tilde{\omega}_{1}^{2}, \tilde{\omega}_{2}^{2}\right\}=\left\{\omega^{2}, \omega^{2}+2 \Omega^{2}\right\} .
\end{gathered}
$$

The normalized ground-state wave function, when chosen as the target state, becomes

$\psi_{T}=\psi_{0}\left(\tilde{x}_{1}\right) \psi_{0}\left(\tilde{x}_{2}\right)=\frac{\left(\tilde{\omega}_{1} \tilde{\omega}_{2}\right)^{1 / 4}}{\sqrt{\pi}} \exp \left[-\frac{1}{2}\left(\tilde{\omega}_{1} \tilde{x}_{1}^{2}+\tilde{\omega}_{2} \tilde{x}_{2}^{2}\right)\right]$. 
We can express this wave function in terms of the physical positions of the two masses:

$\psi_{T}=\frac{\left(\omega_{1} \omega_{2}-\beta^{2}\right)^{1 / 4}}{\sqrt{\pi}} \exp \left[-\frac{\omega_{1}}{2} x_{1}^{2}-\frac{\omega_{2}}{2} x_{2}^{2}-\beta x_{1} x_{2}\right]$,

where

$$
\omega_{1}=\omega_{2}=\frac{1}{2}\left(\tilde{\omega}_{1}+\tilde{\omega}_{2}\right), \quad \beta \equiv \frac{1}{2}\left(\tilde{\omega}_{1}-\tilde{\omega}_{2}\right) .
$$

The above Gaussian wave functions [Eq. (2.8)] are the target state. The reference state, as that in Ref. [15], is chosen to be the following factorized Gaussian state in which the two masses are unentangled,

$$
\psi_{R}=\sqrt{\frac{\omega_{0}}{\pi}} \exp \left[-\frac{\omega_{0}}{2}\left(x_{1}^{2}+x_{2}^{2}\right)\right]
$$

where $\omega_{0}$ is a free parameter that characterizes our reference state. This means that we will compute the complexity of the interacting ground states relative to the noninteracting ground states.

After choosing the reference and target states we have to find a unitary transformation $U$ that implements $\psi_{T}=$ $U \psi_{R}$. In the wave function approach the transformation is considered to relate to the exponential part in the target state wave function [Eq. (2.8)] and in the reference state wave function [Eq. (2.10)]; i.e.,

$$
\omega_{0}\left(x_{1}^{2}+x_{2}^{2}\right) \rightarrow \omega_{1} x_{1}^{2}+\omega_{2} x_{2}^{2}+2 \beta x_{1} x_{2} .
$$

We can use the basic vector $\psi_{R}^{T}=\left(\sqrt{\omega_{0}} x_{1}, \sqrt{\omega_{0}} x_{2}\right)$ to express the above transformation in a matrix form:

$$
\begin{aligned}
\psi_{R}^{T} \cdot \psi_{R} & \rightarrow\left(\psi_{R}^{T} U^{T}\right) \cdot\left(U \psi_{R}\right)=\psi_{R}^{T} \cdot \mathrm{M}_{\mathrm{w}} \cdot \psi_{R}, \\
\mathrm{M}_{\mathrm{w}} & =U^{T} U=\left(\begin{array}{cc}
\frac{\omega_{1}}{\omega_{0}} & \frac{\beta}{\omega_{0}} \\
\frac{\beta}{\omega_{0}} & \frac{\omega_{2}}{\omega_{0}}
\end{array}\right),
\end{aligned}
$$

in which $\mathrm{M}_{\mathrm{w}}$ denotes the transformation matrix in the wave function approach.

Gate matrix $U(s)$ belongs to group $G L(2, R)=$ $R \times S L(2, R)$ which can be expressed as a [15]

$$
U_{G L(2, R)}(s)=e^{y(s)}\left(\begin{array}{cc}
\cos \tau(s) \cosh \rho(s)-\sin \theta(s) \sinh \rho(s) & -\sin \tau(s) \cosh \rho(s)+\cos \theta(s) \sinh \rho(s) \\
\sin \tau(s) \cosh \rho(s)+\cos \theta(s) \sinh \rho(s) & \cos \tau(s) \cosh \rho(s)+\sin \theta(s) \sinh \rho(s)
\end{array}\right)
$$

Regarding the operator $\mathcal{O}^{I}$ in Eq. (1.1) as a group of generators of matrix $M^{I}$, Eq. (1.1) has the solution

$$
Y^{I}(s)=\frac{1}{\operatorname{Tr}\left(\left(M^{J}\right)^{T} M^{J}\right)} \operatorname{Tr}\left(\partial_{s} U(s) U(s)^{-1}\left(M^{I}\right)^{T}\right) .
$$

The line element on the group manifold becomes

$$
\begin{aligned}
d s^{2}= & \delta_{I J} d Y^{I}(s) d Y^{I}(s) \\
= & 2\left(d y^{2}+d \rho^{2}+\cosh (2 \rho) \cosh ^{2} \rho d \tau^{2}\right. \\
& \left.+\cosh (2 \rho) \sinh ^{2} \rho d \theta^{2}-\sinh ^{2}(2 \rho) d \tau d \theta\right),
\end{aligned}
$$

in which the metric $g_{I J}=\delta_{I J}$ is that chosen in Ref. [15]. The geodesics on the above Riemann space were analyzed and it was found that the square of the geodesic length becomes

$$
\begin{aligned}
\text { Distance }^{2} & =\int_{0}^{1} d s \delta_{I J} \frac{d Y^{I}(s)}{d s} \frac{d Y^{I}(s)}{d s} \\
& =2 y(1)+2 \rho(1) .
\end{aligned}
$$

Note that the geodesic solution is that with initial condition $\left((y(0), \rho(0), \tau(0), \theta(0))=\left(0,0,0, \theta_{0}\right)\right.$. While $\theta_{0}$ is undetermined due to the rotation symmetry in the group manifold, we can choose $\theta(0)=\theta(1)=\theta_{0}=0$ in general.
Also, the solution found in Ref. [15] shows that $\tau(s)$ is constant along the geodesic trajectory. Thus $\tau(1)=\tau(0)=1$ and it does not contribute to the geodesic length. The other parts of the geodesic trajectory solutions are $y(s)=y(1) \cdot s$ and $\rho(s)=\rho(1) \cdot s$.

Using Eq. (2.12) the solution of $U^{T}(1) U(1)=\mathrm{M}_{\mathrm{w}}$ is

$$
y(1)=\frac{1}{4} \ln \frac{\tilde{\omega}_{1} \tilde{\omega}_{2}}{\omega_{0}^{2}}, \quad \rho(1)=\frac{1}{4} \ln \frac{\tilde{\omega}_{2}}{\tilde{\omega}_{1}} ;
$$

thus, the geodesic distance in the manifold of Riemann space of group $G L(2, R)$ is

$$
\begin{aligned}
D^{2} & \left.=2(y(1))^{2}+\rho(1)^{2}\right)=\frac{1}{4}\left(\left(\ln \frac{\tilde{\omega}_{1}}{\omega_{0}}\right)^{2}+\left(\ln \frac{\tilde{\omega}_{2}}{\omega_{0}}\right)^{2}\right) \\
& =(\ln \alpha)^{2}+(\ln \gamma)^{2} .
\end{aligned}
$$

Above is that described by Jefferson and Myers in Ref. [15].

The method relies on the Gaussian type wave functions in Eqs. (2.8) and (2.10) in which the quantum gate is a $G L(2, R)$ matrix in Eq. (2.13) and the basic vectors are $\left(\sqrt{\omega_{0}} x_{1}, \sqrt{\omega_{0}} x_{2}\right)$. The gate matrix is used to transform the exponential part of the wave functions from the reference state to the target state. 
The method had been extended to study $\lambda \Phi^{4}$ [24]. In the leading order of small $\lambda$ the ground state wave functions of the target state and the reference state can be expressed as the exponential type. For example, the reference state wave function is $\Psi \sim \exp \left[-\frac{\omega}{2}\left(x_{1}^{2}+x_{2}^{2}+\lambda\left(x_{1}^{4}+x_{2}^{4}+x_{1}^{2} x_{2}^{2}\right)\right)\right]$. Now the basic vectors are $\left(x_{1}, x_{2}, x_{1} x_{2}, x_{1}^{2}, x_{2}^{2}\right)$ and the quantum gate becomes a $G L(5, R)$ matrix. While the wave function becomes more complex it can be studied along the free scalar field case since the wave function is a pure exponential type.

In the cases of the excited harmonic states the wave functions are not described by the pure exponential form. For example, the wave function of the first excited state of the harmonic state is $x e^{-\frac{1}{2} \omega x^{2}}$, which is not a pure exponential form, and then it is hard to work in the wave function approach.

In the following sections we will turn to the operator approach which needs not the explicit function form of a wave function and can be used to study the complexity in the excited states.

\section{COMPLEXITY IN THE GROUND STATE OF 2 HARMONIC OSCILLATORS}

\section{A. Basic scheme}

In the second quantization the target ground state $|0,0\rangle_{\text {target }}$ and the reference ground state state $|0,0\rangle_{\text {ref }}$ are defined by

$$
\tilde{a}_{1} \tilde{a}_{2}|0,0\rangle_{\text {target }}=0, \quad a_{1} a_{2}|0,0\rangle_{\text {ref }}=0,
$$

where

$\tilde{a}_{1}=\sqrt{\frac{\tilde{\omega}_{1}}{2}} \tilde{x}_{1}-i \frac{1}{\sqrt{2 \tilde{\omega}_{1}}} \tilde{p}_{1}, \quad \tilde{a}_{2}=\sqrt{\frac{\tilde{\omega}_{2}}{2}} \tilde{x}_{2}-i \frac{1}{\sqrt{2 \tilde{\omega}_{2}}} \tilde{p}_{2}$, $a_{1}=\sqrt{\frac{\omega_{0}}{2}} x_{1}-i \frac{1}{\sqrt{2 \omega_{0}}} p_{1}, \quad a_{2}=\sqrt{\frac{\omega_{0}}{2}} x_{2}-i \frac{1}{\sqrt{2 \omega_{0}}} p_{2}$.

The relations between these operators are

$\tilde{a}_{1}=\frac{1}{2 \sqrt{2}}\left(\left(\alpha+\alpha^{-1}\right)\left(a_{2}+a_{1}\right)+\left(\alpha-\alpha^{-1}\right)\left(a_{2}^{\dagger}+a_{1}^{\dagger}\right)\right)$,

$\tilde{a}_{2}=\frac{1}{2 \sqrt{2}}\left(\left(\gamma+\gamma^{-1}\right)\left(-a_{2}+a_{1}\right)+\left(\gamma-\gamma^{-1}\right)\left(-a_{2}^{\dagger}+a_{1}^{\dagger}\right)\right)$,

where

$$
\alpha=\sqrt{\frac{\tilde{\omega}_{1}}{\omega_{0}}}, \quad \gamma=\sqrt{\frac{\tilde{\omega}_{2}}{\omega_{0}}} .
$$

We see that both of operators $\tilde{a}_{1}$ and $\tilde{a}_{2}$ depend on the four kinds operators $a_{1}^{\dagger}, a_{1}, a_{2}^{\dagger}, a_{2}$. Thus to consider the transformation between target state operators and reference state operators we have to consider the $4 \times 4$ matrix in the below:

$$
\left(\begin{array}{c}
\tilde{a}_{1}^{\dagger} \\
\tilde{a}_{2}^{\dagger} \\
\tilde{a}_{1} \\
\tilde{a}_{2}
\end{array}\right)=\tilde{\mathrm{M}}_{\mathrm{op}}^{(0)}\left(\begin{array}{c}
a_{1}^{\dagger} \\
a_{2}^{\dagger} \\
a_{1} \\
a_{2}
\end{array}\right)
$$

where

$$
\tilde{\mathrm{M}}_{\mathrm{op}}^{(0)}=\frac{1}{2 \sqrt{2}}\left(\begin{array}{cccc}
\alpha+\alpha^{-1} & \alpha+\alpha^{-1} & \alpha-\alpha^{-1} & \alpha-\alpha^{-1} \\
\gamma+\gamma^{-1} & -\left(\gamma+\gamma^{-1}\right) & \gamma-\gamma^{-1} & -\left(\gamma-\gamma^{-1}\right) \\
\alpha-\alpha^{-1} & \alpha-\alpha^{-1} & \alpha+\alpha^{-1} & \alpha+\alpha^{-1} \\
\gamma-\gamma^{-1} & -\left(\gamma-\gamma^{-1}\right) & \gamma+\gamma^{-1} & -\left(\gamma+\gamma^{-1}\right)
\end{array}\right)
$$

is the gate matrix of the ground state in the operator approach.

Through a $S O(4)$ transformation by a matrix

$$
A_{S O(4)}=\left(\begin{array}{cccc}
\frac{1}{\sqrt{2}} & 0 & \frac{1}{\sqrt{2}} & 0 \\
0 & \frac{1}{\sqrt{2}} & 0 & \frac{1}{\sqrt{2}} \\
\frac{1}{\sqrt{2}} & 0 & -\frac{1}{\sqrt{2}} & 0 \\
0 & \frac{1}{\sqrt{2}} & 0 & -\frac{1}{\sqrt{2}}
\end{array}\right)
$$


the gate matrix $M_{\mathrm{op}}^{(0)}$ becomes a block form, i.e., $\tilde{\mathrm{M}}_{\mathrm{op}}^{(0)} \rightarrow$ $\mathrm{M}_{\mathrm{op}}^{(0)} \equiv A_{S O(4)}^{T} \tilde{\mathrm{M}}_{\mathrm{op}}^{(0)} A_{S O(4)}$, where

$$
\begin{aligned}
\mathrm{M}_{\mathrm{op}}^{(0)} & =\left(\begin{array}{cc}
M_{1} & 0 \\
0 & M_{2}
\end{array}\right), \quad \mathrm{M}_{1}=\frac{1}{\sqrt{2}}\left(\begin{array}{cc}
\alpha & \alpha \\
\gamma & -\gamma
\end{array}\right), \\
\mathrm{M}_{2} & =\frac{1}{\sqrt{2}}\left(\begin{array}{cc}
\alpha^{-1} & \alpha^{-1} \\
\gamma^{-1} & -\gamma^{-1}
\end{array}\right)
\end{aligned}
$$

and

$$
\left(\begin{array}{c}
\tilde{a}_{1}^{\dagger}+\tilde{a}_{1} \\
\tilde{a}_{2}^{\dagger}+\tilde{a}_{2} \\
\tilde{a}_{1}^{\dagger}-\tilde{a}_{1} \\
\tilde{a}_{2}^{\dagger}-\tilde{a}_{2}
\end{array}\right)=\mathrm{M}_{\mathrm{op}}^{(0)}\left(\begin{array}{c}
a_{1}^{\dagger}+a_{1} \\
a_{2}^{\dagger}+a_{2} \\
a_{1}^{\dagger}-a_{1} \\
a_{2}^{\dagger}-a_{2}
\end{array}\right)
$$

Now the gate matrix becomes $G L(2, R) \times G L(2, R)$ and we can use the $G L(2, R)$ matrix representation in Eq. (2.13) to parametrize the matrix $\mathrm{M}_{1}$ and $\mathrm{M}_{2}$.

For the matrix $\mathrm{M}_{1}$ we find that

$$
y(1)=\frac{1}{4} \ln \frac{\tilde{\omega}_{1} \tilde{\omega}_{2}}{\omega_{0}^{2}}, \quad \rho(1)=\frac{1}{4} \ln \frac{\tilde{\omega}_{2}}{\tilde{\omega}_{1}} .
$$

It is interesting to see that the values are exactly those in Eq. (2.17) despite $M_{w} \neq M_{1}$. Thus the geodesic length is just that in the wave function approach.

For the matrix $\mathrm{M}_{2}$

$$
y(1)=-\frac{1}{4} \ln \frac{\tilde{\omega}_{1} \tilde{\omega}_{2}}{\omega_{0}^{2}}, \quad \rho(1)=-\frac{1}{4} \ln \frac{\tilde{\omega}_{2}}{\tilde{\omega}_{1}},
$$

which gives the same geodesic length as that from $\mathrm{M}_{1}$.

At first sight, the total geodesic length from $G L(2, R) \times$ $G L(2, R)$ will be double comparing to that in the wave function approach. In fact, to consider the transformation in the operator approach, for completeness, we had used four operators: $\tilde{a}_{1}^{\dagger}, \tilde{a}_{2}^{\dagger}, \tilde{a}_{1}$, and $\tilde{a}_{2}$, not just two operators, $\tilde{a}_{1}^{\dagger}$ and $\tilde{a}_{2}^{\dagger}$, which create the target state. Thus, to count the proper number of the quantum gate we have to reduce the summation by $\frac{1}{2}$ and

$$
\begin{aligned}
D_{\text {ground state }}^{2} & =\frac{1}{2}\left(D_{M_{1}}^{2}+D_{M_{2}}^{2}\right) \\
& =\frac{1}{4}\left(\left(\ln \frac{\tilde{\omega}_{1}}{\omega_{0}}\right)^{2}+\left(\ln \frac{\tilde{\omega}_{2}}{\omega_{0}}\right)^{2}\right) \\
& =(\ln \alpha)^{2}+(\ln \gamma)^{2} .
\end{aligned}
$$

In this way the complexity from the operator approach fits to that from the wave function approach in Eq. (2.18). Note that the $\mathrm{SO}(4)$ transformation matrix $A_{S O(4)}$ in Eq. (3.9) is just the rotation in the manifold of group $G L(2, R) \times G L(2, R)$ and does not change the geodesic length.

\section{B. Simple scheme}

While the above algorithm is reasonable we will improve it to a simple scheme that is used to evaluate the complexity of any excited states $|n, m\rangle$ in Sec. IV. First we make a linear combination of the Eq. (3.11) and transform it to

$$
\left(\begin{array}{c}
A_{1}^{+} \\
A_{1}^{-} \\
A_{2}^{+} \\
A_{2}^{-}
\end{array}\right)=\mathrm{M}_{\mathrm{op}}\left(\begin{array}{c}
X \\
Y \\
W \\
Z
\end{array}\right)=\left(\begin{array}{cccc}
\alpha & 0 & 0 & 0 \\
0 & \alpha^{-1} & 0 & 0 \\
0 & 0 & \gamma & 0 \\
0 & 0 & 0 & \gamma^{-1}
\end{array}\right)\left(\begin{array}{c}
X \\
Y \\
Z \\
W
\end{array}\right),
$$

where

$$
\begin{aligned}
A_{1}^{+} & =\tilde{a}_{1}^{\dagger}+\tilde{a}_{1}, \quad A_{1}^{-}=\tilde{a}_{1}^{\dagger}-\tilde{a}_{1}, \\
A_{2}^{+} & =\tilde{a}_{2}^{\dagger}+\tilde{a}_{2}, \quad A_{2}^{-}=\tilde{a}_{2}^{\dagger}-\tilde{a}_{2}, \\
X & =\frac{1}{\sqrt{2}}\left(\left(a_{1}^{\dagger}+a_{1}\right)+\left(a_{2}^{\dagger}+a_{2}\right)\right), \\
Y & =\frac{1}{\sqrt{2}}\left(\left(a_{1}^{\dagger}-a_{1}\right)+\left(a_{2}^{\dagger}-a_{2}\right)\right), \\
Z & =\frac{1}{\sqrt{2}}\left(\left(a_{1}^{\dagger}+a_{1}\right)-\left(a_{2}^{\dagger}+a_{2}\right)\right) \\
W & =\frac{1}{\sqrt{2}}\left(\left(a_{1}^{\dagger}-a_{1}\right)-\left(a_{2}^{\dagger}-a_{2}\right)\right) .
\end{aligned}
$$

In this representation $\left(A_{1}^{ \pm}, A_{2}^{ \pm}\right)$are the four target operators and $(X, Y, Z, W)$ are four reference operators. The gate matrix $\mathrm{M}_{\mathrm{op}}$ is diagonal with element $\left(\alpha, \alpha^{-1}, \gamma, \gamma^{-1}\right)$. We emphasize that the diagonal property of the gate matrix in this representation plays a crucial role in calculating the complexity in the $n$th excited state.

We consider first the ground state system in which the target state is defined by $\tilde{a}_{1} \tilde{a}_{2}|0,0\rangle_{\text {target }}=0$. Although it needs only an annihilation operator, here we need also the associated creation operator to form the basic state (operator) in studying the matrix transformation between the reference state and target state, as that mentioned before. This means that in the new representation we need both operators $A_{1}^{+}, A_{1}^{-}$, which relate to $a_{1}^{\dagger}, a_{1}$, and need both operators $A_{2}^{+}, A_{2}^{-}$, which relate to $a_{2}^{\dagger}, a_{2}$. Therefore the basic operator to represent $a_{1}^{\dagger} \tilde{a}_{2}^{\dagger}$ is $\left(A_{1}^{+}, A_{1}^{-}\right) \otimes\left(A_{2}^{+}, A_{2}^{-}\right)$.

Now,

$$
\begin{aligned}
& \left(A_{1}^{+}, A_{1}^{-}\right) \otimes\left(A_{2}^{+}, A_{2}^{-}\right) \\
& =\left(\begin{array}{c}
A_{1}^{+} A_{2}^{+} \\
A_{1}^{+} A_{2}^{-} \\
A_{1}^{-} A_{2}^{+} \\
A_{1}^{-} A_{2}^{-}
\end{array}\right)=\left(\begin{array}{cccc}
\alpha \gamma & 0 & 0 & 0 \\
0 & \alpha \gamma^{-1} & 0 & 0 \\
0 & 0 & \alpha^{-1} \gamma & 0 \\
0 & 0 & 0 & \alpha^{-1} \gamma^{-1}
\end{array}\right)\left(\begin{array}{c}
X Z \\
X W \\
Y Z \\
Y W
\end{array}\right) .
\end{aligned}
$$


We see that above gate matrix is diagonal and we can follow the formula (A7) to evaluate the associated geodesic length. The result is

$$
\begin{aligned}
D_{(0,0)}^{2}= & \frac{1}{4} \cdot\left((\ln (\alpha \gamma))^{2}+\left(\ln \left(\alpha \gamma^{-1}\right)\right)^{2}+\left(\ln \left(\alpha^{-1} \gamma\right)\right)^{2}\right. \\
& \left.+\left(\ln \left(\alpha^{-1} \gamma^{-1}\right)\right)^{2}\right) \\
= & (\ln \alpha)^{2}+(\ln \gamma)^{2} .
\end{aligned}
$$

In this way the complexity calculated in the operator approach fits to the wave function approach in Eq. (2.18). Before we close this section we make two comments about the simple scheme.

1. Normalization factor: Note that we add a normalization factor $\frac{1}{4}$ in Eq. (3.20). This is because to find the gate matrix transformation in the operator approach we use two operators: $A_{1}^{+}, A_{1}^{-}$to relate to $\tilde{a}_{1}$ and $A_{2}^{+}, A_{2}^{-}$to relate to $\tilde{a}_{2}$. Thus the vector space $\left(A_{1}^{+}, A_{1}^{-}\right) \otimes\left(A_{2}^{+}, A_{2}^{-}\right)$, which has 4 elements, is 4 times the original space with operators $\tilde{a}_{1} \tilde{a}_{2}$, which has only one element.

When extending our scheme to the general state $|n, m\rangle_{\text {target }}$, which satisfies the relation

$$
\tilde{a}_{1}^{n+1} \tilde{a}_{2}^{m+1}|n, m\rangle_{\text {target }}=0, \quad n, m \geq 0
$$

it is easy to see that we have to add a normalization factor

Normalization factor of $|n, m\rangle_{\text {target }}=\frac{1}{2^{n+1} 2^{m+1}}$

to obtain the correct value of complexity in state $|n, m\rangle_{\text {target }}$. Below, we always add this normalization factor to calculate the complexity in the excited states.

2. Reference state: Note that in the wave function approach the target state is the interacting harmonic oscillators which, in terms of the norm mode become the state $|0,0\rangle_{\text {target }}$ with frequencies $\tilde{\omega}_{1}$ and $\tilde{\omega}_{2}$. The reference state is $|0,0\rangle_{\text {ref }}$, which is two noninteracting harmonic oscillators with the same frequency $\omega_{0}$. Since $\tilde{a}_{1} \tilde{a}_{2}|0,0\rangle_{\text {target }}=0$ while $a_{1} a_{2}|0,0\rangle_{\text {ref }}=0$ we see that the target operator is $\tilde{a}_{1}, \tilde{a}_{2}$ while the reference operator is $a_{1}$, $a_{2}$ in the operator approach. If turning off the interaction then $\alpha=\frac{\tilde{\omega}_{1}}{\tilde{\omega}_{0}}=\gamma=\frac{\tilde{\omega}_{2}}{\tilde{\omega}_{0}}=1$ and the relations in Eqs. (3.4) and (3.5) become

$$
\begin{aligned}
& \tilde{a}_{1} \stackrel{\alpha=\gamma=1}{\longrightarrow} \frac{1}{\sqrt{2}}\left(a_{1}+a_{2}\right) \nsim a_{1}, \\
& \tilde{a}_{2} \stackrel{\alpha=\gamma=1}{\longrightarrow} \frac{1}{\sqrt{2}}\left(a_{1}-a_{2}\right) \nsim a_{2} .
\end{aligned}
$$

Using Eq. (3.3) we see that $\left(a_{1} \pm a_{2}\right)$ can be regarded as the annihilation operators at position $x_{1} \pm x_{2}$. This means that the reference state is 2 noninteracting harmonic oscillator states $|0,0\rangle_{\text {ref }}$ in which the first and second oscillators are at position $x_{1}+x_{2}$ and $x_{1}-x_{2}$, respectively. After turning on the interaction the target state is 2 interacting harmonic oscillators $|0,0\rangle_{\text {target }}$. Thus, for the target state $|n, m\rangle_{\text {target }}$, which will be studied below, the corresponding reference state is $|n, m\rangle_{\text {ref }}$.

\section{COMPLEXITY IN THE EXCITED STATES OF 2 HARMONIC OSCILLATORS}

\section{A. Examples}

To study the case of excited states let us begin with the case of the ground state.

(i) Ground state $|0,0\rangle_{\text {target }}$ :

The ground state is defined by $\tilde{a}_{1} \tilde{a}_{2}|0,0\rangle_{\text {target }}=0$. From the argument in Sec. III, i.e., the relations $\tilde{a}_{1} \sim A_{1}^{+}+A_{1}^{-}$ and $\tilde{a}_{2} \sim A_{2}^{+}+A_{2}^{-}$, we see that to find the gate matrix we have to consider the following relation:

$$
\begin{aligned}
& \left(A_{1}^{+}+A_{1}^{-}\right)\left(A_{2}^{+}+A_{2}^{-}\right) \\
& \quad=\left(\alpha X+\alpha^{-1} Y\right)\left(\gamma Z+\gamma^{-1} W\right) \\
& \quad=\alpha \gamma X Z+\alpha \gamma^{-1} X W+\alpha^{-1} \gamma Y Z+\alpha^{-1} \gamma^{-1} Y W .
\end{aligned}
$$

We see that the coefficients in last relation, i.e., $\alpha \gamma, \alpha \gamma^{-1}, \alpha^{-1} \gamma, \alpha^{-1} \gamma^{-1}$, are just those in Eq. (3.20), which are used to calculate the complexity.

(ii) Excited state $|1,0\rangle_{\text {target }}$ :

For the excited state defined by $\left(\tilde{a}_{1}\right)^{2} \tilde{a}_{2}|1,0\rangle_{\text {target }}=0$ we have to consider

$$
\begin{aligned}
\left(A_{1}^{+}\right. & \left.+A_{1}^{-}\right)^{2}\left(A_{2}^{+}+A_{2}^{-}\right) \\
= & \left(\alpha X+\alpha^{-1} Y\right)^{2}\left(\gamma Z+\gamma^{-1} W\right) \\
= & \alpha^{2} \gamma X^{2} Z+\alpha^{2} \gamma^{-1} X^{2} W+2 \gamma X Y Z+2 \gamma^{-1} X Y W \\
& +\alpha^{-2} \gamma Y W+\alpha^{-2} \gamma^{-1} Y^{2} W .
\end{aligned}
$$

The coefficients in the last relation are used to calculate the complexity.

(iii) Excited state $|2,0\rangle_{\text {target }}$ :

For the excited state defined by $\left(\tilde{a}_{1}\right)^{3} \tilde{a}_{2}|2,0\rangle_{\text {target }}=0$ we have to consider

$$
\begin{aligned}
\left(A_{1}^{+}\right. & \left.+A_{1}^{-}\right)^{3}\left(A_{2}^{+}+A_{2}^{-}\right)=\left(\alpha+\alpha^{-1}\right)^{3}\left(\gamma+\gamma^{-1}\right) \\
= & \alpha^{3} \gamma+3 \alpha \gamma+3 \alpha^{-1} \gamma+\alpha^{-3} \gamma+\alpha^{3} \gamma^{-1}+3 \alpha \gamma^{-1} \\
& +3 \alpha^{-1} \gamma^{-1}+\alpha^{-3} \gamma^{-1},
\end{aligned}
$$

in which we let $X=Y=Z=W=1$ for simplicity. The coefficients in the last relation are used to calculate the complexity.

(iv) Excited state $|1,1\rangle_{\text {target }}$ : 
For the excited state defined by $\left(\tilde{a}_{1}\right)^{2}\left(\tilde{a}_{2}\right)^{2}|1,1\rangle_{\text {target }}=0$ we have to consider

$$
\begin{aligned}
\left(A_{1}^{+}+\right. & \left.A_{1}^{-}\right)^{2}\left(A_{2}^{+}+A_{2}^{-}\right)^{2}=\left(\alpha+\alpha^{-1}\right)^{2}\left(\gamma+\gamma^{-1}\right)^{2} \\
= & \alpha^{2} \gamma^{2}+\alpha^{2} \gamma^{-2}+\alpha^{-2} \gamma^{2}+\alpha^{-2} \gamma^{-2}+2 \alpha^{2} \\
& +2 \gamma^{2}+2 \alpha^{-2}+2 \gamma^{-2}+4 .
\end{aligned}
$$

The coefficients in the last relation are used to calculate the complexity. Note that the constant term does not depend on $\alpha, \gamma$ and contributes null to the geodesic.

Using the above coefficients in each excited state, which are associated to the matrix element of quantum gate, we can calculate the geodesic length through a simple replacement rule

$$
c \cdot \alpha^{\mathrm{k}} \gamma^{\mathrm{m}} \rightarrow c \cdot[\mathrm{k} \ln \alpha+\mathrm{m} \ln \gamma]^{2}
$$

for any constant value of $c{ }^{1}$ This replacement rule has been used in previous sections and is checked in Eq. (A7). Then we quickly find that

$$
\begin{gathered}
D_{(0,0)}^{2}=(\ln \alpha)^{2}+(\ln \gamma)^{2}, \\
D_{(1,0)}^{2}=2(\ln \alpha)^{2}+(\ln \gamma)^{2}, \\
D_{(2,0)}^{2}=3(\ln \alpha)^{2}+(\ln \gamma)^{2}, \\
D_{(1,1)}^{2}=2(\ln \alpha)^{2}+2(\ln \gamma)^{2},
\end{gathered}
$$

after imposing the proper normalization factor $\frac{1}{2^{n+1} 2^{m+1}}$ mentioned in Eq. (3.23).

We can easily extend the calculations to higher excited states. The results indicate that the square of the length of the quantum circuit in the general state $|n, m\rangle_{\text {target }}$ is $D_{(\mathrm{n}, \mathrm{m})}^{2}=(n+1)(\ln \alpha)^{2}+(m+1)(\ln \gamma)^{2}$. We show this formula below.

\section{B. General formula}

Using the previous arguments, the property of the gate matrix in the state $|n-1, m-1\rangle_{\text {target }}$ is read from the coefficients in the following expansion:

$$
\begin{aligned}
\left(\alpha+\alpha^{-1}\right)^{n}\left(\gamma+\gamma^{-1}\right)^{m} & =\sum_{i, j, k, \ell \geq 0}^{n, m}(\alpha)^{i}\left(\alpha^{-1}\right)^{j}(\gamma)^{k}\left(\gamma^{-1}\right)^{\ell} C_{i j k \ell}^{n, m} \\
& =\sum_{i, j, k, \ell \geq 0}^{n, m} \alpha^{i-j} \gamma^{k-\ell} C_{i j k \ell}^{n, m}, \quad n, m \geq 1,
\end{aligned}
$$

\footnotetext{
${ }^{1}$ Constant $c$ represents the multiplicity in which $\alpha^{\mathrm{k}} \gamma^{\mathrm{m}}$ appears. It is the coefficient $C_{i j k \ell}^{n}$ in Eq. (4.10).
}

where the summation is constrained by $i+j=n, k+\ell=m$. $C_{i j k \ell}^{n, m}$ are the coefficients in the expansion. To calculate the geodesic length from the above coefficients we can use the replacement rule in Eq. (4.5). Thus,

$$
\begin{aligned}
D_{(\mathrm{n}-1, \mathrm{~m}-1)}^{2}= & \frac{1}{2^{n} 2^{m}} \sum_{i, j, k, \ell \geq 0}^{n, m}[(i-j) \ln \alpha+(k-\ell) \ln \gamma]^{2} C_{i j k \ell}^{n, m} \\
= & \frac{1}{2^{n} 2^{m}}\left(\sum_{i, j, k, \ell \geq 0}^{n, m}(i-j)^{2} C_{i j k \ell}^{n, m}\right)(\ln \alpha)^{2} \\
& +\frac{1}{2^{n} 2^{m}}\left(\sum_{i, j, k, \ell \geq 0}^{n, m}(k-\ell)^{2} C_{i j k \ell}^{n, m}\right)(\ln \gamma)^{2} \\
= & n(\ln \alpha)^{2}+m(\ln \gamma)^{2}, \quad n, m \geq 1
\end{aligned}
$$

where we have used the summation formulas in Eqs. (B5) and (B6).

\section{Wave function of the excited states}

In this subsection we will make a comment about the wave function of the excited state in this paper. For the simple harmonic oscillator we have the well-known properties

$$
\begin{aligned}
H & =\hbar \omega\left(a_{\omega}^{\dagger} a_{\omega}\right), \quad H\left|n_{\omega}\right\rangle=\hbar \omega\left(n_{\omega}+\frac{1}{2}\right)\left|n_{\omega}\right\rangle, \\
\left|n_{\omega}\right\rangle & =\frac{\left(a_{\omega}^{\dagger}\right)^{n_{\omega}}}{\sqrt{n_{\omega} !}}|0\rangle .
\end{aligned}
$$

Therefore, while $|0\rangle$ is the ground (vacuum) state we can regard $\left|n_{\omega}\right\rangle$ as the $n$th excited state. On the other hand, in occupation number representation the state $\left|n_{\omega}\right\rangle$ can also be represented, for example, as

$$
\left|n_{\omega}\right\rangle=|\overbrace{1_{\omega}, \ldots, 1_{\omega}}^{n_{\omega}}\rangle .
$$

Consider, for example, the excited state $\left|2_{\omega_{a}}, 1_{\omega_{b}}\right\rangle$, which can be regarded as the system with two oscillators, $N=2$-one of which is the second excited state $\left(\Psi_{\omega_{a}}^{(2 \text { th })}\right)$ with frequency $\omega_{a}$ and another is the first excited state $\left(\Psi_{\omega_{b}}^{(1 \text { th })}\right)$ with frequency $\omega_{b}$. The corresponding wave function in the first quantization is

$$
\frac{1}{\sqrt{2}}\left(\Psi_{\omega_{a}}^{(2 \mathrm{th})}\left(x_{1}\right) \Psi_{\omega_{b}}^{(1 \mathrm{th})}\left(x_{2}\right)+\Psi_{\omega_{b}}^{(1 \mathrm{th})}\left(x_{1}\right) \Psi_{\omega_{a}}^{(2 \mathrm{th})}\left(x_{2}\right)\right)
$$

On the other hand, using the relation (4.13) we find another representation $\left|2_{\omega_{a}}, 1_{\omega_{a}}\right\rangle=\left|1_{\omega_{a}}, 1_{\omega_{a}}, 1_{\omega_{b}}\right\rangle$ and the corresponding wave function in the first quantization is 


$$
\begin{aligned}
& \frac{1}{\sqrt{3}}\left(\Psi_{\omega_{a}}^{(1 \text { th })}\left(x_{1}\right) \Psi_{\omega_{a}}^{(1 \text { th })}\left(x_{2}\right) \Psi_{\omega_{b}}^{(1 \text { th })}\left(x_{3}\right)\right. \\
& \quad+\Psi_{\omega_{a}}^{(1 \text { th })}\left(x_{1}\right) \Psi_{\omega_{b}}^{(1 \text { th })}\left(x_{2}\right) \Psi_{\omega_{a}}^{(1 \text { th })}\left(x_{3}\right) \\
& \left.\quad+\Psi_{\omega_{b}}^{(1 \text { th })}\left(x_{1}\right) \Psi_{\omega_{a}}^{(1 \text { th })}\left(x_{2}\right) \Psi_{\omega_{a}}^{(1 \text { th })}\left(x_{3}\right)\right)
\end{aligned}
$$

In this representation there are three oscillators, $N=3$, and two among them have the same frequency $\omega_{a}$.

Now, both the $N=2$ wave function [Eq. (4.14)] and the $N=3$ wave function [Eq. (4.15)] could be used to represent state $\left|2_{\omega_{a}}, 1_{\omega_{b}}\right\rangle$ and, at first sight, there is ambiguity in choosing a wave function to represent $\left|2_{\omega_{a}}, 1_{\omega_{b}}\right\rangle$ in our calculation. In fact, from Eq. (5.5) we see that the frequencies of the two oscillators, $N=2$, is different from the frequencies of the three oscillators, $N=3$. Therefore, after using Eq. (5.5) to choose the proper frequencies, $\left(\omega_{a}, \omega_{b}\right)$, of the $N=2$ oscillators, the wave function in Eq. (4.14) can be used to represent the state $\left|2_{\omega_{a}}, 1_{\omega_{b}}\right\rangle$. In this case, the $N=3$ wave function in Eq. (4.15) could not be used to represent the state $\left|2_{\omega_{a}}, 1_{\omega_{b}}\right\rangle$ since the frequencies $\left(\omega_{a}, \omega_{a}, \omega_{b}\right)$ do not fit the relation (5.5).

Therefore, the state $\left|n_{\omega_{a}}, m_{\omega_{b}}\right\rangle$ considered in this paper is the system with 2 oscillators in which one is at the $n$th excited state with frequency $\omega_{a}$ while another is at the $m$ th excited state with frequency $\omega_{b}$. The corresponding wave function in the first quantization is

$$
\begin{aligned}
\left|n_{\omega_{a}}, m_{\omega_{b}}\right\rangle \rightarrow & \frac{1}{\sqrt{2}}\left(\Psi_{\omega_{a}}^{(\mathrm{nth})}\left(x_{1}\right) \Psi_{\omega_{b}}^{(\mathrm{mth})}\left(x_{2}\right)\right. \\
& \left.+\Psi_{\omega_{b}}^{(\mathrm{mth})}\left(x_{1}\right) \Psi_{\omega_{a}}^{(\mathrm{nth})}\left(x_{2}\right)\right) .
\end{aligned}
$$

The arguments can be applied to many oscillator systems.

\section{COMPLEXITY IN $N$ HARMONIC OSCILLATORS AND LATTICE SCALAR FIELD THEORY}

The above calculations are performed on the 2 coupled harmonic oscillators and, for self-consistency, in this section we will describe how these calculations can be extended to scalar field theory.

The lattice version of the one-dimensional free scalar field can be described by one-dimensional $N$ harmonic oscillators. The Hamiltonian is that extended 2 coupled harmonic oscillator in Eq. (2.4) to $N$ harmonic oscillators

$$
H=\frac{1}{2} \sum_{k=1}^{N} p_{k}^{2}+\omega^{2} x_{k}^{2}+\Omega^{2}\left(x_{k}-x_{k+1}\right)^{2}
$$

with periodic boundary condition $x_{k+N+1}=x_{k}$ [15]. In the normal coordinate the Hamiltonian becomes

$$
H=\frac{1}{2} \sum_{k=1}^{N} \tilde{p}_{k}^{2}+\tilde{\omega}_{k}^{2} \tilde{x}_{k}^{2}
$$

where [15]

$$
\begin{gathered}
\tilde{x}_{k}=\frac{1}{\sqrt{N}} \sum_{j=1}^{N} \exp \left(\frac{-2 \pi i k}{N} j\right) x_{j}, \\
\tilde{p}_{k}=\frac{1}{\sqrt{N}} \sum_{j=1}^{N} \exp \left(\frac{-2 \pi i k}{N} j\right) p_{j}, \\
\tilde{\omega}_{k}^{2}=\omega^{2}+4 \Omega^{2} \sin ^{2} \frac{\pi k}{N} .
\end{gathered}
$$

In the operator approach we need the relations between the annihilation operators in original coordinates and in normal coordinates. The conventional definitions are

$\tilde{a}_{k}=\sqrt{\frac{\tilde{\omega}_{k}}{2}} x_{k}-i \frac{1}{\sqrt{2 \tilde{\omega}_{k}}} p_{k}, \quad a_{k}=\sqrt{\frac{\omega_{0}}{2}} x_{k}-i \frac{1}{\sqrt{2 \omega_{0}}} p_{k}$,

in which $\omega_{0}$ is the reference state frequency used in Eq. (3.6). The relation between the operators is

$$
\begin{aligned}
\tilde{a}_{k}= & \frac{1}{2 \sqrt{N}}\left(\alpha_{k}+\alpha_{k}^{-1}\right)\left(\sum_{j=1}^{N} \exp \left(\frac{-2 \pi i k}{N} j\right) a_{j}\right) \\
& +\frac{1}{2 \sqrt{N}}\left(\alpha_{k}-\alpha_{k}^{-1}\right)\left(\sum_{j=1}^{N} \exp \left(\frac{-2 \pi i k}{N} j\right) a_{j}^{\dagger}\right),
\end{aligned}
$$

where

$$
\alpha_{k}=\sqrt{\frac{\tilde{\omega}_{k}}{\omega_{0}}}, \quad k=1, \ldots, N .
$$

Following the investigations in previous sections we can now use the following reference operators $\left\{X_{k}^{+}, X_{k}^{-}\right\}$and target operators $\left\{A_{k}^{+}, A_{k}^{-}\right\}$

$$
\begin{aligned}
A_{k}^{+} & \equiv \tilde{a}_{k}^{\dagger}+\tilde{a}_{k}=\alpha_{k}\left(\frac{1}{\sqrt{N}} \sum_{j=1}^{N} \exp \left(\frac{-2 \pi i k}{N} j\right)\left(a_{j}^{\dagger}+a_{j}\right)\right) \\
& \equiv \alpha_{k} X_{k}^{+}, \\
A_{k}^{-} & \equiv \tilde{a}_{k}^{\dagger}-\tilde{a}_{k}=\alpha_{k}^{-1}\left(\frac{1}{\sqrt{N}} \sum_{j=1}^{N} \exp \left(\frac{-2 \pi i k}{N} j\right)\left(a_{j}^{\dagger}-a_{j}\right)\right) \\
& \equiv \alpha_{k}^{-1} X_{k}^{-} .
\end{aligned}
$$

In this representation the gate matrix is diagonal. 
Now we can follow the investigation in the previous section to calculate the complexity of state $\left|n_{1} \cdots n_{N}\right\rangle$ in lattice scalar field theory. The extension of Eq. (4.10) tells us that we shall consider the following expansion:

$$
\begin{aligned}
\prod_{k=1}^{N}\left(\alpha_{k}+\alpha_{k}^{-1}\right)^{n_{k}} & =\sum_{\left\{i_{k} \geq 0, j_{k} \geq 0\right\}}^{\left\{n_{k}\right\}} \prod_{k=1}^{N}\left(\alpha_{k}\right)^{i_{k}}\left(\alpha_{k}^{-1}\right)^{j_{k}} C_{\left\{i_{k}, j_{k}\right\}}^{\left\{n_{k}\right\}} \\
& =\sum_{\left\{i_{k} \geq 0, j_{k} \geq 0\right\}} \prod_{k=1}^{N}\left(\alpha_{k}\right)^{i_{k}-j_{k}} C_{\left\{i_{k}, j_{k}\right\}}^{\left\{n_{k}\right\}},
\end{aligned}
$$

where the summation is constrained by $i_{k}+j_{k}=n_{k}$. $C_{\left\{i_{k}, j_{k}\right\}}^{\left\{n_{k}\right\}}$ are the coefficients in the expansion. To calculate the geodesic length from the above coefficients we can use the replacement rule in Eq. (4.5). Thus,

$$
\begin{aligned}
D_{\left(\left\{\mathrm{n}_{k}-1\right\}\right)}^{2} & =\frac{1}{2^{n_{1}+\cdots+n_{N}}} \sum_{\left\{i_{k} \geq 0, j_{k} \geq 0\right\}}^{n}\left[\sum_{k=1}^{N}\left(i_{k}-j_{k}\right) \ln \left(\alpha_{k}\right)\right]^{2} C_{\left\{i_{k}, j_{k}\right\}}^{\left\{n_{k}\right\}} \\
& =\frac{1}{2^{n_{1}+\cdots+n_{N}}} \sum_{k=1}^{N}\left(\sum_{\left\{i_{k} \geq 0, j_{k} \geq 0\right\}}^{\left\{n_{k}\right\}}\left(i_{k}-j_{k}\right)^{2} C_{\left\{i_{k}, j_{k}\right\}}^{\left\{n_{k}\right\}}\right) \ln \left(\alpha_{k}\right)
\end{aligned}
$$

$$
=\sum_{k=1}^{N} n_{k} \ln \left(\alpha_{k}\right)
$$

where we have used the summation formula in Eq. (B11). Above result is the extension of Eq. (4.11) which is the case of $N=2$.

The extension to the $d-1$ dimensional coupled harmonic oscillation (which describes the $d$ dimensional free scalar field theory) is just to replace above $k$ by $\vec{k}=\left(k_{1}, k_{2}, \ldots, k_{d-1}\right)$, as that described in Ref. [15]. The result is

$$
D_{\left(\left\{n_{k_{\mathrm{i}}}-1\right\}\right)}^{2}=\sum_{i=1}^{d-1} \sum_{k=1}^{N} n_{k_{i}} \ln \left(\alpha_{k_{i}}\right), \quad \alpha_{k_{i}}=\sqrt{\frac{\tilde{\omega}_{k_{i}}}{\omega_{0}}}
$$

which is the excited-state complexity of the $d$ dimensional free scalar field theory on lattice.

\section{DISCUSSIONS}

In this paper we present the operator approach to calculate the complexity of the free scalar field. We first quickly review the method in Ref. [15] and describe the lattice free scalar field as coupled harmonic oscillators. Then, as a first step, we investigate the 2 coupled oscillators and regard the transformation between the creation operators in the reference state and target state as the gate matrix. We find that the gate matrix can be reduced to diagonal form after orthogonal transformation, then we calculate the complexity of the ground state. The result reproduces the known value of ground-state complexity in Ref. [15]. While the excited-state wave function of harmonic oscillation is not the pure exponential form and the wave function approach is hard to work, the operator approach, which need not use the explicit form of the wave function, can be used to study the complexity in the excited state. We explicitly calculate the complexity in several excited states and present a simple derivation to find the general formula of complexity in any excited states. Finally, we describe how the calculations in the two coupled harmonic oscillators can be extended to $N$ coupled harmonic oscillators, which describes the lattice free scalar field theory.

Some more studies are necessary to clarify the property of using the operator approach to calculate the complexity:

1. We have calculated the complexity of excited states from field theory. It is interesting to find the holographic results to compare our results. Note that, as that discussed in Sec. III B, the reference state of the ground state is different from that of excited states and there are different ones for each excited state. This is problematic for the holographic motivation of this work. For example, should the growing size of a black hole reflect the relative complexity between a time-dependent microstate and a time-dependent reference? If we allow the reference to be time dependent, then we are essentially introducing a timedependent free parameter into the problem of explaining one time-dependent quantity (the volume or WdW patch action, or whatever), which means we have zero predictability and zero falsifiability.

2. We only study the free scalar field in this paper. The extension to interacting theory and fermion theory is deserving of study.

3. Since the operator approach needs not know the position-space wave function it can be used to study the complexity in the spin system that appears in many models of condensed matter.

These problems are understudied.

\section{ACKNOWLEDGMENTS}

The author is grateful to the anonymous referee for many useful comments which have significantly improved the quality of the paper after several corrections.

\section{APPENDIX A: GEODSIC LENGTH IN EQUIVALENT GATE MATRIX}

Consider the following two gate matrices

$$
M_{1}=\left(\begin{array}{cc}
\alpha & \alpha \\
\gamma & -\gamma
\end{array}\right) ; \quad M_{2}=\left(\begin{array}{cc}
\alpha & 0 \\
0 & \gamma
\end{array}\right)
$$

where parameters $\alpha$ and $\gamma$ could be any value. Since 


$$
M_{1} \cdot\left(\begin{array}{c}
\Psi_{a} \\
\Psi_{b}
\end{array}\right)=M_{2} \cdot\left(\begin{array}{c}
\Psi_{a}+\Psi_{b} \\
\Psi_{a}-\Psi_{b}
\end{array}\right)
$$

we say that matrix $M_{2}$ is the matrix $M_{1}$ after rearrangement (or linear combination).

We can regard both matrices as $G L(2, R)$ and use the function form in Eq. (2.13) to calculate the geodesic lengths. Both give the same geodesic length

$$
\begin{aligned}
D_{G L(2, R)}^{2} & =2\left(\frac{1}{2}(\ln \alpha+\ln \gamma)^{2}\right)+2\left(\frac{1}{2}(\ln \alpha-\ln \gamma)^{2}\right) \\
& =(\ln \alpha)^{2}+(\ln \gamma)^{2} .
\end{aligned}
$$

On the other hand, we can regard the second matrix as $R \times R$ and express it in a general matrix

$$
M_{R \times R}=\left(\begin{array}{cc}
e^{y_{1}} & 0 \\
0 & e_{2}^{y}
\end{array}\right) \quad y_{1}=\ln \alpha, \quad y_{2}=\ln \gamma .
$$

The associated metric and geodesic length are

$$
\begin{aligned}
& d s_{R \times R}^{2}= \operatorname{tr}\left(d e^{y_{1}} e^{-y_{1}}\right) \operatorname{tr}\left(d e^{y_{1}} e^{-y_{1}}\right) \\
&+\operatorname{tr}\left(d e^{y_{2}} e^{-y_{2}}\right) \operatorname{tr}\left(d e^{y_{2}} e^{-y_{2}}\right) \\
&=\left(d y_{1}\right)^{2}+\left(d y_{2}\right)^{2}, \\
& D_{R \times R}^{2}=(\ln \alpha)^{2}+(\ln \gamma)^{2},
\end{aligned}
$$

which is consistent with Eq. (A3).

Therefore for a diagonal gate matrix $M$ the associated geodesic length $D_{M}^{2}$ is

$D_{M}^{2}=\operatorname{diagonal}\left(e^{y_{1}}, \ldots, e^{y_{k}}, \ldots, e^{y_{n}}\right) \Rightarrow D_{M}^{2}=\sum_{k=1}^{n}\left(\ln y_{k}\right)^{2}$.

The relation is extensively used in this paper.

\section{APPENDIX B: SUMMATION FORMULA}

In this Appendix we consider two summation formulas:

(i) Begin with the definition

$$
(a+b)^{n}(c+d)^{m}=\sum_{i, j, k, \ell \geq 0}^{n, m} a^{i} b^{j} c^{k} d^{\ell} C_{i j k \ell}^{n, m},
$$

where the summation is constrained by $i+j=n$, $k+\ell=m . \quad C_{i j k \ell}^{n, m}$ are the coefficients in expansion. Considering three cases of the derivative of the above relation: 1. Derivative one time with respect to $a$. 2. Derivative two times with respect to $a$. 3. Derivative with respect to $a$ then to $b$. Let $a=b=c=d=1$ in the above three kinds of derivatives and we have three relations

$$
n 2^{n-1} 2^{m}=\sum_{i, j, k, \ell \geq 0}^{n, m} i C_{i j k \ell}^{n, m},
$$

$$
\begin{gathered}
n(n-1) 2^{n-1} 2^{m}=\sum_{i, j, k, \ell \geq 0}^{n, m} i(i-1) C_{i j k \ell}^{n, m}, \\
n(n-1) 2^{n-1} 2^{m}=\sum_{i, j, k, \ell \geq 0}^{n, m} i j C_{i j k \ell}^{n, m} .
\end{gathered}
$$

Using the above relations we find a summation formula

$$
\sum_{i, j, k, \ell \geq 0}^{n}(i-j)^{2} C_{i j k \ell}^{n}=n 2^{n} 2^{m} .
$$

In a similar way we can find another formula

$$
\sum_{i, j, k, \ell \geq 0}^{n}(k-\ell)^{2} C_{i j k \ell}^{n}=m 2^{n} 2^{m}
$$

The above two formulas are used to obtain the general formula (4.11).

(ii) Begin with the definition

$$
\begin{aligned}
& \left(a_{1}+b_{1}\right)^{n_{1}} \cdots\left(a_{k}+b_{k}\right)^{n_{k}} \cdots\left(a_{N}+b_{N}\right)^{n_{N}} \\
& =\sum_{\left\{i_{s} \geq 0, j_{s} \geq 0\right\}}^{\left\{n_{s}\right\}} a_{1}^{i_{1}} b_{1}^{j_{1}} \cdots a_{k}^{i_{k}} b_{k}^{j_{k}} \cdots a_{N}^{i_{N}} b_{N}^{j_{N}} C_{\left\{i_{s}, j_{s}\right\}}^{\left\{n_{s}\right\}},
\end{aligned}
$$

where the summation is constrained by $i_{s}+j_{s}=n_{s}$, $s=1,2 \ldots, N . C_{\left\{i_{s}, j_{s}\right\}}^{\left\{n_{n}\right\}}$ are the coefficients in expansion. Consider three cases of the derivative of the above relation: 1. Derivative one time with respect to $a_{k}$. 2. Derivative two times with respect to $a_{k}$. 3. Derivative with respect to $a_{k}$ then to $b_{k}$. Let $a_{1}=b_{1}=\cdots=a_{N}=b_{N}=1$ after above three kinds of derivative we have three relations:

$$
n_{k} \times 2^{-1+\sum_{s} n_{s}}=\sum_{\left\{i_{s} \geq 0, j_{s} \geq 0\right\}}^{\left\{n_{s}\right\}} i_{k} C_{\left\{i_{s}, j_{s}\right\}}^{\left\{n_{s}\right\}}
$$

$n_{k}\left(n_{k}-1\right) \times 2^{-2+\sum_{s} n_{s}}=\sum_{\left\{i_{s} \geq 0, j_{s} \geq 0\right\}}^{\left\{n_{s}\right\}} i_{k}\left(i_{k}-1\right) C_{\left\{i_{s}, j_{s}\right\}}^{\left\{n_{s}\right\}}$,

$$
n_{k}\left(n_{k}-1\right) \times 2^{-2+\sum_{s} n_{s}}=\sum_{\left\{i_{s} \geq 0, j_{s} \geq 0\right\}}^{\left\{n_{s}\right\}} i_{k} j_{k} C_{\left\{i_{s}, j_{s}\right\}}^{\left\{n_{s}\right\}} .
$$

Using above relations we find a summation formula

$$
\sum_{\left\{i_{s} \geq 0, j_{s} \geq 0\right\}}^{\left\{n_{s}\right\}}\left(i_{k}-j_{k}\right)^{2} C_{\left\{i_{s}, j_{s}\right\}}^{\left\{n_{s}\right\}}=2^{\sum_{s} n_{s}} \times n_{k},
$$

which is used to obtain the general formula (5.13). The case of the $N=2$ above relation reduces to Eqs. (B5) and (B6). 
[1] M. van Raamsdonk, Building up spacetime with quantum entanglement, Gen. Relativ. Gravit. 42, 2323 (2010).

[2] B. Swingle and M. Van Raamsdonk, Universality of gravity from entanglement, arXiv:1405.2933.

[3] N. Lashkari, M. B. McDermott, and M. Van Raamsdonk, Gravitational dynamics from entanglement thermodynamics, J. High Energy Phys. 04 (2014) 195.

[4] T. Faulkner, M. Guica, T. Hartman, R. C. Myers, and M. Van Raamsdonk, Gravitation from entanglement in holographic CFTs, J. High Energy Phys. 03 (2014) 051.

[5] S. Ryu and T. Takayanagi, Holographic Derivation of Entanglement Entropy from the anti de Sitter Space/ Conformal Field Theory Correspondence, Phys. Rev. Lett. 96, 181602 (2006).

[6] J. Maldacena, Eternal black holes in anti-de Sitter, J. High Energy Phys. 04 (2003) 021.

[7] T. Hartman and J. Maldacena, Time evolution of entanglement entropy from black hole interiors, J. High Energy Phys. 05 (2013) 014.

[8] D. Carmi, S. Chapman, H. Marrochio, R. C. Myers, and S. Sugishita, On the time dependence of holographic complexity, J. High Energy Phys. 11 (2017) 188.

[9] T. Ali, A. Bhattacharyya, S. S. Haque, E. H. Kim, and N. Moynihan, Time evolution of complexity: A critique of three methods, J. High Energy Phys. 04 (2019) 087.

[10] T. Ali, A. Bhattacharyya, S. S. Haque, E. H. Kim, and N. Moynihan, Post-quench evolution of distance and uncertainty in a topological system: Complexity, entanglement and revivals, arXiv:1811.05985.

[11] L. Susskind, Computational complexity and black hole horizons, Fortschr. Phys. 64, 24 (2016).

[12] A. R. Brown, D. A. Roberts, L. Susskind, B. Swingle, and Y. Zhao, Holographic Complexity Equals Bulk Action?, Phys. Rev. Lett. 116, 191301 (2016).
[13] A. R. Brown, D. A. Roberts, L. Susskind, B. Swingle, and Y. Zhao, Complexity, action, and black holes, Phys. Rev. D 93, 086006 (2016).

[14] S. Chapman, H. Marrochio, and R. C. Myers, Complexity of formation in holography, J. High Energy Phys. 01 (2017) 062.

[15] R. A. Jefferson and R. C. Myers, Circuit complexity in quantum field theory, J. High Energy Phys. 10 (2017) 107.

[16] S. Chapman, M. P. Heller, H. Marrochio, and F. Pastawski, Towards Complexity for Quantum Field Theory States, Phys. Rev. Lett. 120, 121602 (2018).

[17] R. Khan, C. Krishnan, and S. Sharma, Circuit complexity in Fermionic field theory, Phys. Rev. D 98, 126001 (2018).

[18] L. Hackl and R. C. Myers, Circuit complexity for free fermions, J. High Energy Phys. 07 (2018) 139.

[19] J. Jiang and X. Liu, Circuit complexity for Fermionic thermofield double states, Phys. Rev. D 99, 026011 (2019).

[20] D. W. F. Alves and G. Camilo, Evolution of complexity following a quantum quench in free field theory, J. High Energy Phys. 06 (2018) 029.

[21] H. A. Camargo, P. Caputa, D. Das, M. P. Heller, and R.Jefferson, Complexity as a Novel Probe of Quantum Quenches: Universal Scalings and Purifications, Phys. Rev. Lett. 122, 081601 (2019).

[22] J. Jiang, J. Shan, and J. Yang, Circuit complexity for free Fermion with a mass quench, arXiv:1810.00537.

[23] M. Guo, J. Hernandez, R. C. Myers, and S. M. Ruan, Circuit complexity for coherent states, J. High Energy Phys. 10 (2018) 011.

[24] A. Bhattacharyya, A. Shekar, and A. Sinha, Circuit complexity in interacting QFTs and RG flows, J. High Energy Phys. 10 (2018) 140. 